\title{
Dióxido de estanho nanoestruturado como sensor de $\mathrm{NO}_{\mathrm{x}}$ (Nanostructured tin dioxide as a $\mathrm{NO}_{x}$ gas sensor)
}

\author{
A. P. Maciel, F. Paro, E. R. Leite, E. Longo \\ Centro Multidisciplinar de Desenvolvimento de Materiais Cerâmicos - CMDMC/LIEC/DQ \\ Universidade Federal de S. Carlos \\ Rod. Washington Luiz, km 235, C. P. 676, S. Carlos, SP, 13565-905 \\ adeilton@liec.ufscar.br
}

\begin{abstract}
Resumo
Neste trabalho, nanopartículas de $\mathrm{SnO}_{2}$ foram obtidas pelo método do precursor polimérico e caracterizadas por difração de raios X, isotermas de adsorção-dessorção, microscopia eletrônica de varredura e microscopia eletrônica de transmissão. Apenas a fase cassiterita (tetragonal) foi observada. O material obtido apresenta com alta área superficial e porosidade. Estas características são pré-requisitos para um bom sensor de gás. A sensibilidade ao $\mathrm{NO}_{x}$ para o $\mathrm{SnO}_{2}$ foi estudado na faixa de temperatura compreendida entre 200 e 500 ${ }^{\circ} \mathrm{C}$. Observou-se uma baixa sensibilidade entre 200 e $350^{\circ} \mathrm{C}$, porém, a partir de $400^{\circ} \mathrm{C}$ ocorreu um aumento de três vezes na sensibilidade do sensor. A máxima sensibilidade ocorreu em $400{ }^{\circ} \mathrm{C}$, com um tempo de resposta de $730 \mathrm{~s}$
\end{abstract}

Palavras-chave: nanopartículas, $\mathrm{SnO}_{2}$, sensores de gases.

\section{Abstract}

In this work $\mathrm{SnO}_{2}$ nanoparticles were obtained by the polimeric precursor method and characterized by X-ray diffraction, gas adsorption and desorption isotherms, scanning electron microscopy and transmission electron microscopy. Only the cassiterite (tetragonal) phase was observed. The obtained material presents a high surface area and high porosity. These characteristics are prerequisites for a good gas sensor. The $N O$ sensibility was studied in the temperature range between 200 and $500{ }^{\circ} \mathrm{C}$. A low sensibility between 200 to $350{ }^{\circ} \mathrm{C}$ is also observed; however, starting from $400{ }^{\circ} \mathrm{C}$, an increase of three times in the sensor sensibility occurs. The maximum sensibility was measured at $400{ }^{\circ} \mathrm{C}$ with a response time of $730 \mathrm{~s}$.

Keywords: nanoparticles, $\mathrm{SnO}_{2}$, gas sensors.

\section{INTRODUÇÃOO}

O dióxido de estanho $\left(\mathrm{SnO}_{2}\right)$ e o óxido de zinco $(\mathrm{ZnO})$ são os dois compostos mais usados como sensores resistivos para gases. $\mathrm{O} \mathrm{SnO}_{2}$, além de ser um bom elemento sensor, vem sendo aplicado em muitos campos por apresentar algumas propriedades características, tais como alta condutividade elétrica, alta transparência na região do visível, alta estabilidades térmica, mecânica e química. As aplicações tecnológicas deste material incluem o desenvolvimento de células solares, dispositivos óptico-eletrônicos, displays de cristal líquido, catalisadores, e sensor de gases [1-9], sendo esta última uma das principais aplicações deste material.

Em 1952, Weisz [10] publicou um trabalho envolvendo o fenômeno de barreira eletrônica em óxidos durante a adsorção de espécies nas suas superfícies. No ano seguinte, foi publicado o estudo do efeito de transferência de carga entre o adsorbato e o sólido na quimissorção [11]. Entretanto, somente em 1962, uma década depois, é que os óxidos semicondutores foram aplicados como sensores de gases [12].

Os sensores de gases baseados em materiais semicondutores são amplamente usados nos mais diversos ambientes (domésticos, comerciais, indústrias, etc.), para detecção e/ou monitoramento de gases inflamáveis ou tóxicos, assim como gases produzidos por combustão, oxidação ou decomposição de materiais. A sensibilidade do sensor de dióxido de estanho está intimamente ligada à mudança de sua condutividade elétrica, resultante da interação química ou física entre os gases e a sua superfície [13-17]. O desenvolvimento de sensores deste tipo é muito importante para muitas aplicações, devido suas vantagens, tais como tamanho reduzido, alta estabilidade, sensibilidade e longa vida útil.

Várias pesquisas estão concentradas nos mais diferentes métodos de obtenção de $\mathrm{SnO}_{2}$ nanocristalino, principalmente com o intuito de melhorar as suas propriedades físico-químicas. Entre os métodos mais empregados pode-se citar coprecipitação, sol-gel, condensação de fase gasosa, "spraypirólise", micro-emulsão, microondas, oxidação de estanho metálico, precursor polimérico e rotas hidrotérmicas, entre outras [18-24]. As técnicas de co-precipitação e sol-gel são, geralmente, preferidas por apresentarem bons resultados e serem mais fáceis de implementar.

O método que vem sendo bastante utilizado para obtenção de nanopartículas de dióxido de estanho, em especial $\mathrm{SnO}_{2}$ dopado, é o do precursor polimérico (Pechini) [25]. No presente trabalho é apresentado um estudo das propriedades sensoras de partículas nanométricas de $\mathrm{SnO}_{2}$, obtidas pelo método do precursor polimérico, para detecção de $\mathrm{NO}_{\mathrm{x}}$. 


\section{MATERIAIS E MÉTODOS}

Para obtenção das nanopartículas de dióxido de estanho partiu-se do citrato de estanho, que foi preparado com base na síntese realizada por Besso [26]. A resina de estanho foi obtida a partir da dissolução do citrato de estanho em etilenoglicol, na razão 1:1 em massa. Para que se obtenha uma completa dissolução do citrato de estanho, foi gotejado ácido nítrico concentrado que facilitou a polimerização e a dissolução. A temperatura foi elevada até aproximadamente $120^{\circ} \mathrm{C}$, para eliminação de $\mathrm{NO}_{\mathrm{x}}$.

$\mathrm{O}$ pó do material nanoestruturado foi preparado a partir da pirólise da resina, por 2 horas, a $350{ }^{\circ} \mathrm{C}$. O material carbonizado obtido da pirólise foi desagregado em almofariz de ágata e peneirado (100 mesh). Em seguida, o carvão foi calcinado a $400{ }^{\circ} \mathrm{C}$, por $4 \mathrm{~h}$, para eliminação completa do material orgânico. Em continuidade, o material foi tratado termicamente na temperatura de $550{ }^{\circ} \mathrm{C}$, por $2 \mathrm{~h}$. Os compactos cerâmicos (pastilhas) foram preparados a partir da prensagem uniaxial dos pós. Utilizou-se um molde de $9 \mathrm{~mm}$ de diâmetro e pressão de $210 \mathrm{MPa}$. Depois de prensadas, as amostras foram sinterizadas por duas horas a $550{ }^{\circ} \mathrm{C}$ ao ar.

As análises dos difratogramas de raios $\mathrm{X}$ foram obtidas usando um equipamento da Siemens, modelo D-5000, em configuração $\theta-2 \theta$. Para obtenção das imagens de microscopia eletrônica de transmissão (MET) fez-se uso de um equipamento Philips CM200, operando a 200 kV. Para as imagens de microscopia eletrônica de varredura (MEV) foi utilizado um microscópio eletrônico de varredura Zeiss modelo DSM 940A.

As análises de área superficial específica (BET) e distribuição de tamanho de poros (BJH) foram feitas num equipamento Micrometrics ASAP 2000.

As medidas das propriedades sensoras do dióxido de estanho foram realizadas numa câmara com volume fixo de 1,4 L. A temperatura na câmara é controlada por dispositivo Gefram 500. O controle da pressão na câmara é feito por um sensor Pirani Balzers modelo TPR250, os fluxos dos gases são controlados por válvulas solenóides [27]. Os valores de resistência foram obtidos num multímetro digital Hewlett Packard, modelo 34401A, com limite máximo de detecção entre dois pontos de $1,2 \times 10^{8} \Omega$.

Para realização dos experimentos foram seguidas as etapas: inicialmente fez-se vácuo na câmara, em seguida as amostras foram expostas a ciclos alternados de fluxo de ar sintético e da mistura ar/ $\mathrm{NO}_{x}$. O ciclo é composto de um fluxo de ar sintético de $669 \mathrm{~mL} \cdot \mathrm{min}^{-1}$ por $10 \mathrm{~min}$, seguido de um fluxo misto de ar sintético e $\mathrm{NO}_{\mathrm{x}}$, também de $4 \mathrm{~mL} \cdot \mathrm{min}^{-1}$ por 5 min. Ao término de cada ciclo foram tomados os valores das resistências. Após 10 min. com ar sintético, tem-se $R_{a r}$, e após 5 min em exposição ao $\mathrm{NO}_{x}$, tem-se $R_{g}$; a sensibilidade do sensor (S) foi determinada usando-se a equação abaixo.

$$
S\left|\frac{R_{a r} \quad R_{g}}{R_{a r}}\right| .100
$$

\section{RESULTADOS E DISCUSSÃO}

As nanopartículas de $\mathrm{SnO}_{2}$, na forma de pastilha sinterizada a $550{ }^{\circ} \mathrm{C}$ por $2 \mathrm{~h}$, apresentam apenas a fase cassiterita (tetragonal), Fig. 1.

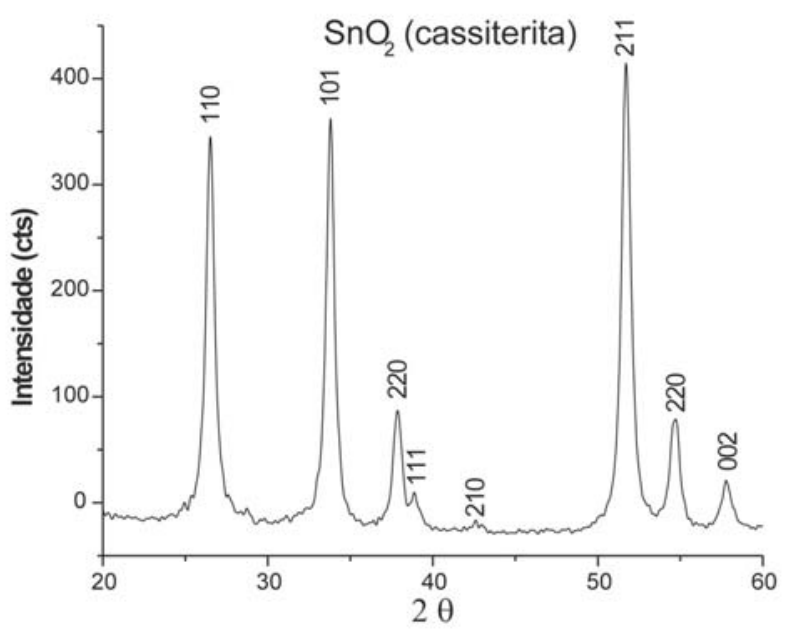

Figura 1: DRX da pastilha de $\mathrm{SnO}_{2}$ sinterizada a $550{ }^{\circ} \mathrm{C}$ por $2 \mathrm{~h}$. [Figure 1: XDR patterns of the ceramic compact $\mathrm{SnO}_{2}$ annealed at $550{ }^{\circ} \mathrm{C} / 2$ h.]
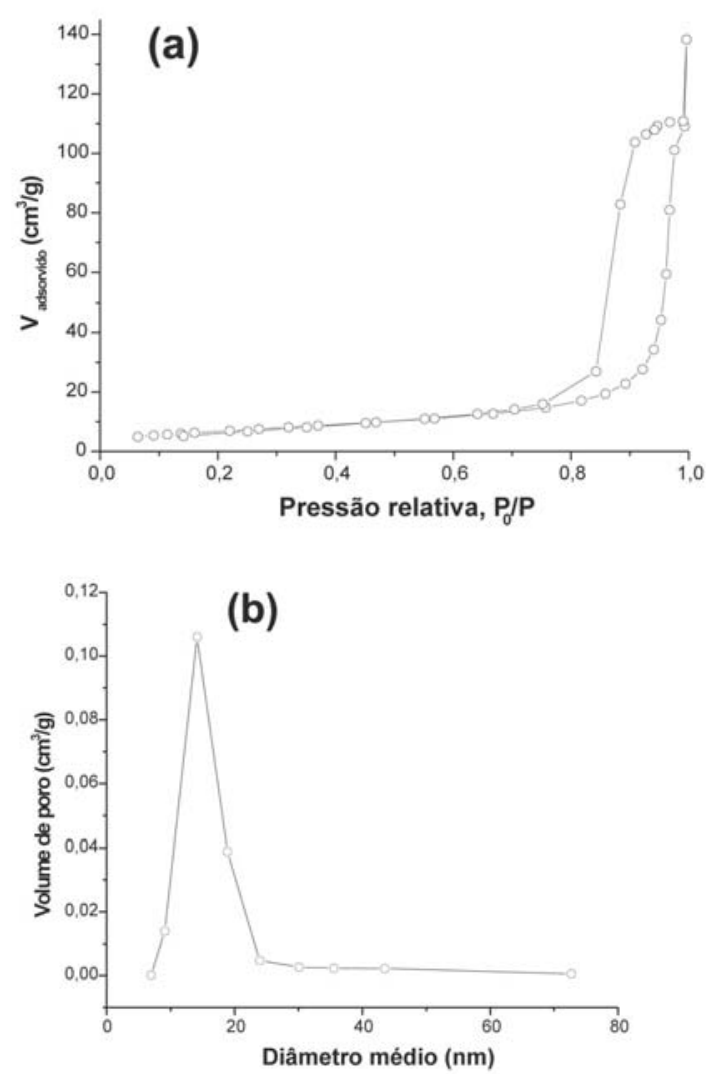

Figura 2: Isoterma de adsorção-dessorção (a) e distribuição de tamanhos de poros (b) da pastilha de $\mathrm{SnO}_{2}$ sinterizada a $550{ }^{\circ} \mathrm{C}$ por $2 \mathrm{~h}$.

[Figure 2: Adsorption-desorption isotherm (a) and pore size distribution (b) of the $\mathrm{SnO}_{2}$ ceramic compact annealed at $550^{\circ} \mathrm{C} / 2 \mathrm{~h}$.] 
Por meio da técnica de BET [28], observa-se que este material apresenta isoterma de adsorção do tipo IV e histerese de adsorção/ dessorção do tipo H1, Fig. 2a, que é típica de materiais que contém poros cilíndricos abertos. Pode ser observada a presença de um ponto destacado em altos valores de $\mathrm{P} / \mathrm{P}_{0}$, relacionado à condensação do gás fora dos poros. A pastilha de $\mathrm{SnO}_{2}$ puro apresenta uma distribuição de tamanhos de poros, obtido pelo método BJH [29], mais freqüente em $18 \mathrm{~nm}$ de diâmetro médio, Fig. 2b, e apresenta uma área superficial de $24 \mathrm{~m}^{2} / \mathrm{g}$.

Observa-se nas micrografias MEV desta pastilha, Fig. 3a, a presença de grandes aglomerados, com cerca de $10 \mu \mathrm{m}$ de diâmetro, composto de outros aglomerados menores, Fig. 3b. Desta forma, o material apresenta dois tipos de poros, um tipo originado entre os aglomerados e outro entre as partículas.

As nanopartículas formadas apresentam-se na forma de aglomerados cristalinos, compostos de cristalitos com diâmetro médio de $12,7 \mathrm{~nm}$. Na Fig. 4 é ilustrada a imagem de MET de baixo aumento das nanopartículas de $\mathrm{SnO}_{2}$.

A avaliação das propriedades sensoras do $\mathrm{SnO}_{2}$ obtido pelo método do precursor polimérico foi realizada numa atmosfera
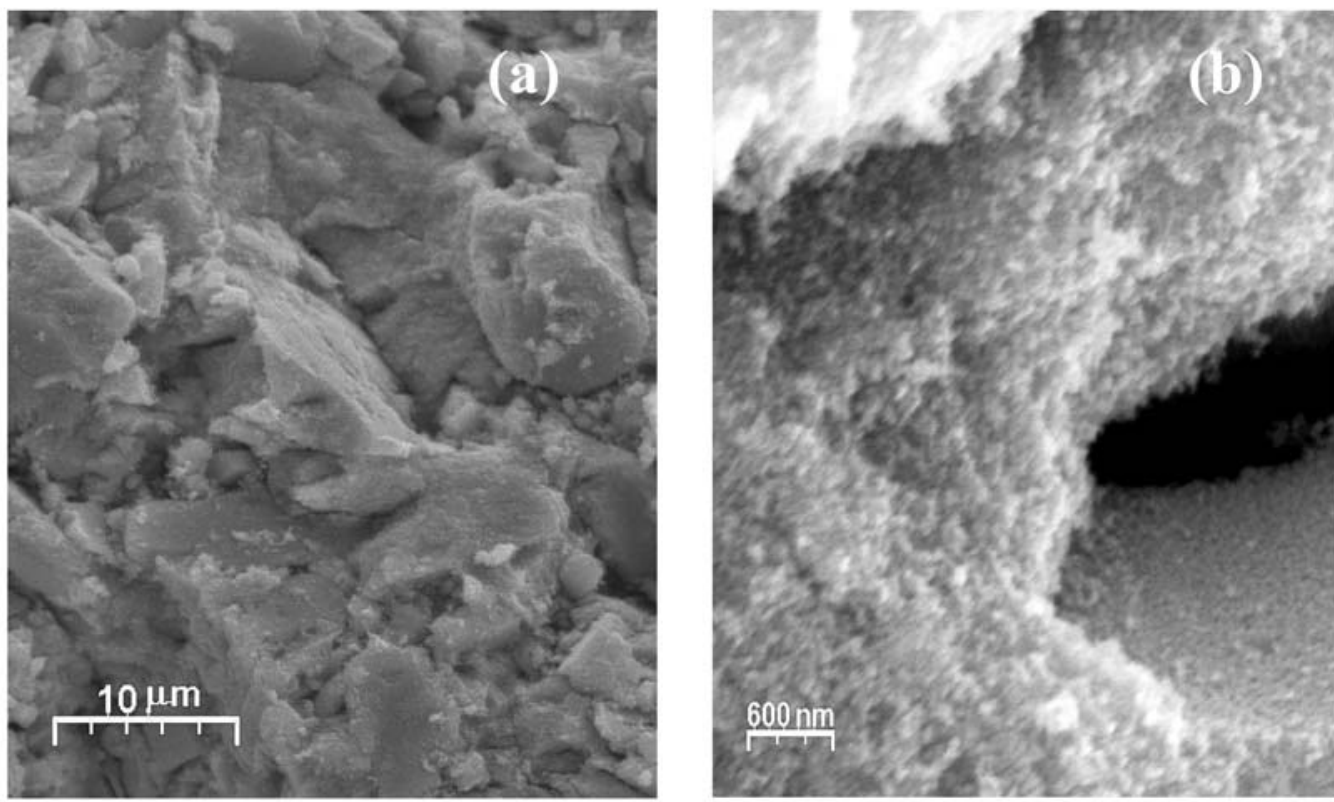

Figura 3: Micrografias obtidas por microscopia eletrônica de varredura da pastilha de $\mathrm{SnO}_{2}$ sinterizada a $550{ }^{\circ} \mathrm{C}$ por $2 \mathrm{~h}$. [Figure 3: SEM micrographs of the $\mathrm{SnO}_{2}$ ceramic compact annealed at $550{ }^{\circ} \mathrm{C} / 2 \mathrm{~h}$.]
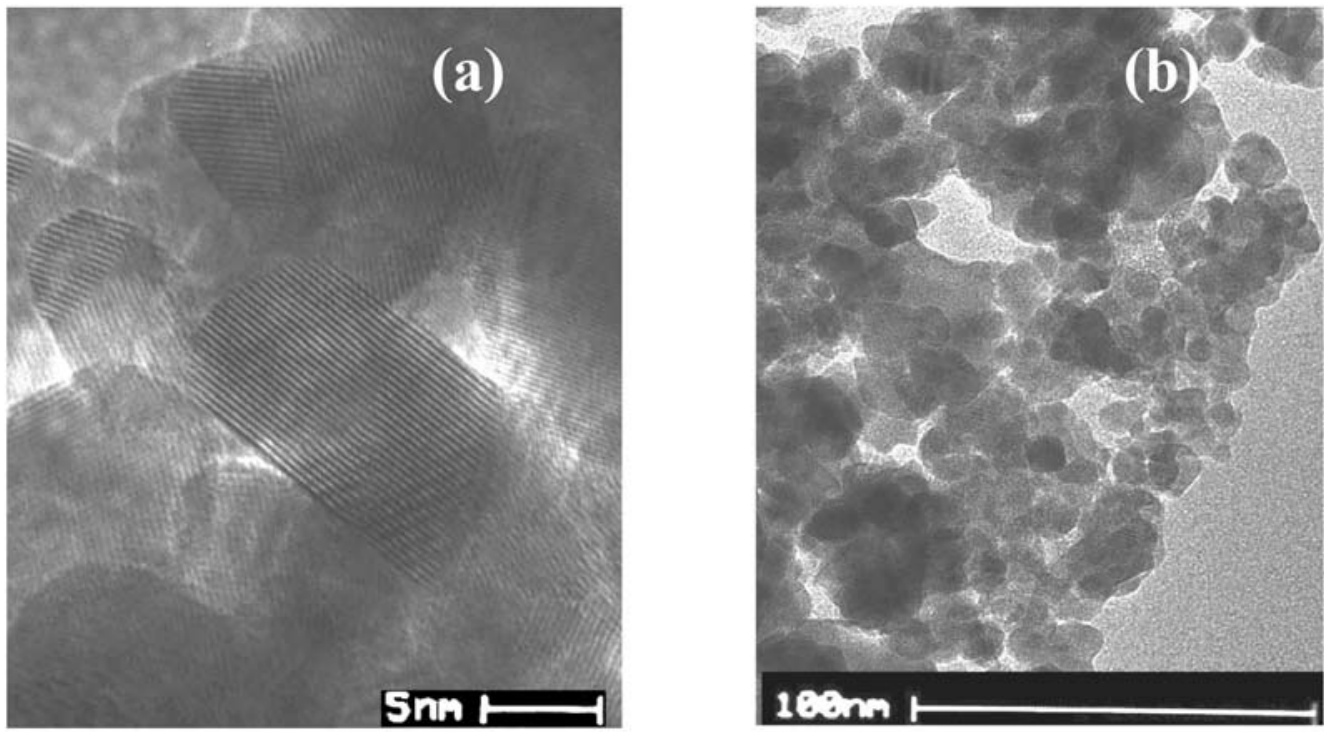

Figura 4: Imagem obtida por microscopia eletrônica de transmissão de alta resolução (a) e baixo aumento (b) das nanopartículas de $\mathrm{SnO}_{2}$.

[Figure 4: TEM high resolution images (a) and low magnification (b) of the $\mathrm{SnO}_{2}$ nanoparticles.] 
de 62 ppm de $\mathrm{NO}_{x}$, sob fluxo de ar sintético.

Uma das principais características dos sensores resistivos para gases baseados em óxidos semicondutores é que a sensibilidade ao gás varia consideravelmente com a temperatura. Deste modo, a determinação da temperatura de trabalho é muito importante para que se obtenha uma maior sensibilidade a um dado gás, neste caso $\mathrm{NO}_{x}$. A operação de sensores de $\mathrm{SnO}_{2}$ a temperaturas abaixo de $200{ }^{\circ} \mathrm{C}$ pode reduzir sua vida útil, uma vez que o sensor fica mais susceptível ao envenenamento por gases fortemente oxidantes, como é o caso do $\mathrm{NO}_{x}$. A operação em temperaturas superiores a $500^{\circ} \mathrm{C}$ fica comprometida, porque o número de elétrons promovidos à banda de condução por efeito térmico é bastante elevado, ocasionando interferências nas medidas, a quantidade oxigênio adsorvida na superfície do óxido é reduzida sensivelmente e ainda pode ocorrer à oxidação térmica dos gases a serem analisados [30].

A Fig. 5 ilustra a variação da sensibilidade ao $\mathrm{NO}_{\mathrm{x}} \mathrm{com}$ a temperatura para o $\mathrm{SnO}_{2}$, quando submetido a uma mistura em volume de $0,2 \%$ de $\mathrm{NO}_{x}, 80,0 \%$ ar sintético e $19,8 \%$ de $\mathrm{N}_{2}$ (gás inerte diluente do $\mathrm{NO}_{\mathrm{x}}$ ).

Os resultados das sensibilidades para uma dada temperatura foram obtidos em triplicata. Assim, na Fig. 5 estão apresentados os valores médios para a faixa de temperatura de 200 a $500{ }^{\circ} \mathrm{C}$. Um dos maiores problemas existentes neste tipo de sensores é justamente na reprodutibilidade dos resultados obtidos.

Pode-se observar na Fig. 5 duas regiões bem definidas com respeito à sensibilidade ao $\mathrm{NO}_{\mathrm{x}}$. A primeira exibe valores de sensibilidade mais baixos que os apresentados pela segunda região, que tem início a $400{ }^{\circ} \mathrm{C}$. Ainda observa-se que entre 350 e $400{ }^{\circ} \mathrm{C}$ ocorre um aumento de aproximadamente três vezes na sensibilidade do material, e que a máxima sensibilidade na faixa de temperatura compreendida entre 200 e $500{ }^{\circ} \mathrm{C}$ ocorreu em $400{ }^{\circ} \mathrm{C}$.

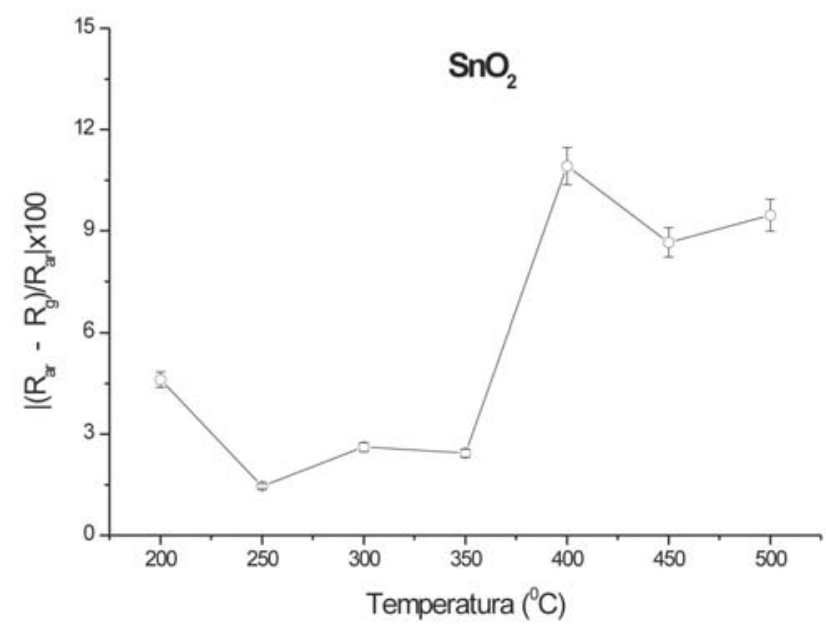

Figura 5: Variação com a temperatura da sensibilidade ao $\mathrm{NO}_{\mathrm{x}}$ para o $\mathrm{SnO}_{2}$.

[Figure 5: $\mathrm{NO}_{x}$ sensibility variation with temperature for $\mathrm{SnO}_{2}$.]

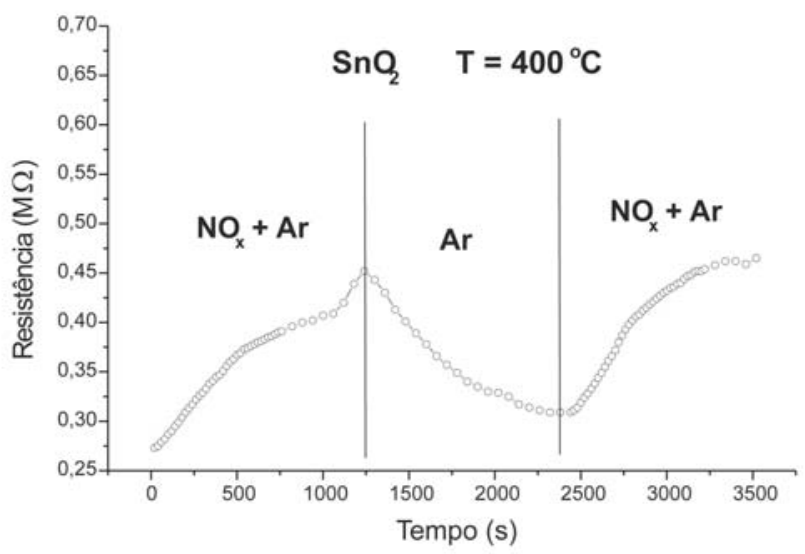

Figura 6: Variação da resistência com o tempo de injeção de $\mathrm{NO}_{x}$ (0,2\% v/v) a $400{ }^{\circ} \mathrm{C}$ para o $\mathrm{SnO}_{2}$.

[Figure 6: Electrical resistance as a function of the $\mathrm{NO}_{x}$ injection time $(0,2 \% \mathrm{v} / \mathrm{v})$ at $400{ }^{\circ} \mathrm{C}$ for $\mathrm{SnO}_{2}$.]

A temperatura escolhida para determinação do tempo de resposta do material sensor foi $400{ }^{\circ} \mathrm{C}$, por ser nesta temperatura que $\mathrm{o} \mathrm{SnO}_{2}$ sintetizado tem maior sensibilidade, como foi descrito anteriormente.

Na Fig. 6 pode-se observar que a resistência inicial a $400{ }^{\circ} \mathrm{C}$, na presença de fluxo de ar sintético, está em aproximadamente $275 \mathrm{k} \Omega$. À medida que $\mathrm{NO}_{\mathrm{x}}$ é adicionado, a resistência elevase até cerca de $450 \mathrm{k} \Omega$, mas na realidade a $1000 \mathrm{~s}$ (resistência igual a $405 \mathrm{k} \Omega$ ) de injeção do gás, já é obtida uma estabilização na resistência da amostra. A injeção de $\mathrm{NO}_{x}$ foi encerrada em 1060 s; no entanto, a câmara ainda ficou carregada com o gás até que este seja arrastado pelo ar, causando um aumento na resistência do material, mesmo depois ter sido cortado o fluxo de gás. Em cerca de 730 s o sistema atinge o valor de $85 \%$ da resposta; deste modo, este valor é considerado como o tempo de resposta do $\mathrm{SnO}_{2}$ frente ao $\mathrm{NO}_{\mathrm{x}}$ a $400{ }^{\circ} \mathrm{C}$.

$\mathrm{Na}$ literatura são encontrados vários valores para o tempo de resposta de sensores de $\mathrm{SnO}_{2}$ para o $\mathrm{NO}_{x}$. Para o sensor de $\mathrm{SnO}_{2}$ dopado com Pt o tempo de resposta é de $1200 \mathrm{~s}$ [31], enquanto que para o sensor de $\mathrm{SnO}_{2}$ dopado com $\mathrm{Cu}$, o tempo de resposta é de $1080 \mathrm{~s}$ [32]. Sensores de $\mathrm{SnO}_{2}$ na forma de filmes finos vem apresentando valores de tempo de resposta próximos de $500 \mathrm{~s}[33,34]$. Este fato fortalece ainda mais as expectativas de que as nanopartículas $\mathrm{SnO}_{2}$ obtidas neste trabalho, depositadas na forma de filmes, possam apresentar excelentes resultados como sensor de gases. Os resultados obtidos mostram que este material, na forma de pastilha, apresenta um bom desempenho como sensor de $\mathrm{NO}_{x}$. Por outro lado, a adição de terras raras como dopantes no $\mathrm{SnO}_{2}$ tem mostrado bons resultados nas propriedades sensoras deste material. Por exemplo, quando o $\mathrm{SnO}_{2}$ foi dopado com nióbio, ocorreu uma diminuição no tamanho de cristalito e uma redução no tempo de resposta em sensores de etanol [27, 35]. Então, dando prosseguimento neste trabalho, serão realizados alguns testes de sensibilidade com o $\mathrm{SnO}_{2}$ dopado com $\mathrm{Ce}, \mathrm{Y}$ e $\mathrm{La}$, os quais apresentam baixa taxa de crescimento a altas temperaturas [36]. 


\section{CONCLUSÕES}

A sensibilidade ao $\mathrm{NO}_{\mathrm{x}}$ para o $\mathrm{SnO}_{2}$ (obtido neste trabalho pelo método do precursor polimérico), na faixa de temperatura compreendida entre 200 e $500{ }^{\circ} \mathrm{C}$, é próxima aos valores obtidos por outros trabalhos. Observa-se entre 200 e $350{ }^{\circ} \mathrm{C}$ uma sensibilidade mais baixa, cerca de um terço, quando comparada à sensibilidade a $400{ }^{\circ} \mathrm{C}$. O tempo de resposta do sensor foi menor do que os apresentados pelo $\mathrm{SnO}_{2}$ obtido por outras técnicas; isto está relacionado ao fato de que este material é poroso e apresenta-se em escala nanométrica.

\section{AGRADECIMENTOS}

Os autores agradecem o suporte financeiro da FAPESP/ CEPID, CAPES, CNPq/PRONEX e FINEP.

\section{REFERÊNCIAS}

[1] E. Llobet, J. Rubio, X. Vilanova, J. Brezmes, X. Corrieg, J. W. Gardner, E. L. Hines, Sens. Actuators B 76 (2001) 419. [2] M. Mwamburi, E. Wackelgard, Solar Energy 68 (2000) 371.

[3] W. Gopel, K. D. Shierbaum, H. D. Wienhofer, Solid State Ionics 28 (1990) 1691.

[4] E. Bucher, Appl. Phys. 17 (1978) 1.

[5] J. G. Fagan, V. R. W. Amarakoon, Am. Ceram. Soc. Bull. 72 (1993) 119.

[6] M. Jarzebski, J. P. Marton, J. Electrochem. Soc. 129 (1976) 299.

[7] J. G. Duhm, J. W. Jou, B. S. Chiou, J. Electrochem. Soc. 136 (1989) 2740.

[8] V. K. Singh, R. Dwivedi, S. K. Srivastava, Microelectronics J. 27 (1996) 531.

[9] L. M. Cukrov, P. G. McCormick, K. Galatsis, W. Wlodarski, Sens. Actuators B 77 (2001) 491.

[10] P. B. Weisz, J. Chem. Phys. 20 (1952) 1483.

[11] P. B. Weisz, J. Chem. Phys. 21 (1953) 1531.

[12] T. Seiyama, A. Kato, K. Fujishi, M. Nagatani, Anal. Chem. 34 (1962) 1502.

[13] N. Yamazoe, Y. Kurokawa, T. Seyama, Sens. Actuators B 4 (1983) 283.

[14] Y. Takao, Y. Iwanaga, Y. Shimisu, M. Egashira, Sens. Actuators B 10 (1993) 229.
[15]H. Yamaura, J. Tamaki, K. Moriya, N. Miura, N. Yamazoe, J. Electrochem. Soc. 143 (1996) L36.

[16]T. Maekawa, J. Tamaki, N. Miura, N. Yamazoe, Chem. Lett. (1991) 575.

[17]M. Akiyama, J. Tamaki, N. Miura, N. Yamazoe, Chem. Lett. (1991) 1611.

[18]E. R. Leite, J. A. Cerri, E. Longo, J. A. Varela, C. A. Paskocimas, J. Eur. Ceram. Soc. 21 (2001) 669.

[19] C. Nayral, T. Ould-Ely, A. Maisonnat, B. Chaudret, P. Fau, L. Lescouzères, A. Peyre-Lavigne, Adv. Mater. 11 (1999) 61. [20]E. R. Leite, I. T. Weber, E. Longo, J. A. Varela, Adv. Mater. 12 (2000) 965.

[21] J. M. Herrmann, J. Disdier, A. Fernández, V. M. Jiménez, J. C. Sánchez-López, Nanoestructured Mater. 8 (1997) 675.

[22] K.C. Song, J. H. Kim, Powder Technology 107 (2000) 268. [23] A. Cirera, A. Vilà, A. Cornet, J. R. Morante, Mater. Sci. Eng. C 15 (2001) 203.

[24]L. B. Fraigi, D. G. Lamas, N. E. Walsöe de Reca, Mater. Lett. 47 (2001) 262.

[25]M. Pechini, US Pat. No. 3330697 (1967).

[26] M. M. Besso, U.S. Pat. No. 3.213.120 (1965).

[27] I. T. Weber, R. Andrade, E. R. Leite, E. Longo, Sens. Actuators B 72 (2001) 180.

[28] S. Brunauer, L. S. Emmet, E. Teller, J. Am. Chem. Soc. 60 (1938) 309.

[29] E. P. Barret, L. G. Joyner, P. P. Halenda, J. Am. Chem. Soc. 73 (1951) 373.

[30] K. D. Schierbaum, U. Weimar, W. Göpel, Sens. Actuators B 27 (1995) 157.

[31] I. Sayago, J. Gutiérrez, L. Ares, J. I. Robla, M. C. Horrilo, J. Getino, J. Rino, J. A. Agapito, Sens. Actuators B 26-27 (1995) 19.

[32] G. Zhang, M. Liu, Sens. Actuators B 69 (2000) 144.

[33.] Karthingeyan, R. P. Gupta, K. Scharnagl, M. Burgmair, M. Zimmer, S. K. Sharma, I. Eisele, Sens. Actuators B 78 (2001) 69.

[34] E. Comini, G. Faglia, G. Sberveglieri, Sens. Actuators B 76 (2001) 270.

[35] I. T. Weber, E. Longo, E. Leite, Mater. Letters 43 (2000) 166.

[36]E. R. Leite, A. P. Maciel, I. T. Weber, P. N. Lisboa-Filho, E. Longo, C. O. Paiva-Santos, A. V. C. Andrade, C. A. Paskocimas, Y. Maniette, W. H. Schreiner, Adv. Mater. 14 (2002) 905.

(Rec. 19/03/03, Ac. 11/07/03) 